\title{
Alzheimer disease risk factor CALM modulates tau turnover
}

CALM (phosphatidylinositol-binding clathrin assembly protein, also known as PICALM), a gene that has been identified as a risk factor for Alzheimer disease $(A D)$, encodes a protein that modulates the autophagy pathway and affects cellular clearance of tau protein, according to new research published in Nature Communications.

Along with the emergence of amyloid plaques in the brain, accumulation of tau in the form of intraneuronal neurofibrillary tangles is a classic neuropathological hallmark of AD.

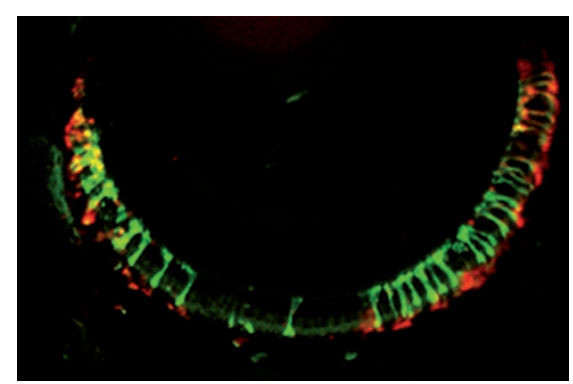

Section of zebrafish retina expressing human tau (green). Rod photoreceptors (red) are lost as a consequence of the tau expression. Image courtesy of D. C. Rubinsztein.
Previous studies have shown that clearance of tau occurs via autophagy, a mechanism that is responsible for sequestering damaged or undesirable cellular components and delivering them to the lysosomal system for degradation.

"We used cell biology approaches to determine if and how altered CALM function impacted on autophagy," explains David Rubinsztein, who led the new study. "In addition, we used Drosophila models to test whether loss of CALM function influenced tau accumulation, and generated novel zebrafish models that enabled us to test whether excess CALM affected tau toxicity and clearance."

The researchers employed an RNA interference approach to knock down CALM expression in various cultured cell lines. They found that loss of CALM function caused impairments in autophagosome formation and autophagosome-lysosome fusion. In a Drosophila model, knockdown of the $C A L M$ homologue lap led to an increase in tau levels.
Interestingly, upregulation of the CALM gene in cell culture and zebrafish models also resulted in impaired autophagy and accumulation of tau. Overexpression of CALM in zebrafish photoreceptors led to degeneration of these cells, probably as a consequence of tau toxicity.

"We have linked an AD disease risk locus to tau accumulation via autophagy, and provide a plausible link between CALM and AD pathology," concludes Rubinsztein. "A key question is whether the CALM locus associated with $\mathrm{AD}$ houses alleles that result in decreased or increased function; we will be interested to follow up and test CALM functional variants that impact on disease risk."

Heather Wood

Original article Moreau, K. et al. PICALM modulates autophagy activity and tau accumulation. Nat. Commun. doi:10.1038/ncomms5998

Further reading Harris, H. \& Rubinsztein, D. C. Control of autophagy as a therapy for neurodegenerative disease. Nat. Rev. Neurol. 8, 108-117 (2012) 